\title{
Does it need to Take Digitally Targeted Biopsies Additionally to TRUS Guided 12- Core Systematic Prostate Biopsies? A Single Center Prospective Clinical Study
}

\author{
Ali Erol1', Sarp Korcan Keskin ${ }^{1 *}$ and Huseyin Akyildiz ${ }^{2}$ \\ ${ }^{1}$ Bahcesehir University, Department of Urology, Turkey \\ ${ }^{2}$ Goztepe Medical Park Hospital, Department of Urology, Turkey
}

*Corresponding author: Sarp Korcan Keskin, Bahcesehir University, Department of Urology, Istanbul, Turkey, Tel: +905359444400; Email: urologum@gmail.com

\section{Research Article}

Volume 4 Issue 1

Received Date: January 28, 2019

Published Date: February 25, 2019

DOI: $10.23880 /$ oajun-16000156

\section{Abstract}

Purpose: To investigate whether digitally targeted additional biopsies enhance the detection rate of standard 12-core TRUS guided systematic biopsies.

Methods: A total of 119 patients who either have a PSA level greater than $4 \mathrm{ng} / \mathrm{ml}$ or suspicious DRE regardless of their PSA level underwent a twelve cores TRUS guided standard systematic prostate biopsies and two additional DRE guided targeted biopsies in case of abnormal DRE. The patients were divided into three groups with regards to their PSA level; Group I ( $\mathrm{n}=8)$ : PSA<4ng/ml, Group II ( $\mathrm{n}=62)$ : PSA 4-10ng/ml and Group III ( $\mathrm{n}=49)$ : PSA>10ng/ml. In each group, the independent diagnostic yield of DRE guided biopsies was compared to the standard systematic biopsies.

\section{The Results:}

Group I: In 3 out of 8 patients in this group were diagnosed as poorly differentiated prostate cancer. DRE guided biopsies detected one cancer out of 2 in this group.

Group II: TRUSG guided systematic biopsies revealed 6 prostate cancer out of 7 cancer cases. DRE guided biopsies were also positive for prostate cancer in only 1 case, while standard 12-core systematic biopsies had failed to depict the cancer.

Group III: Both TRUSG guided systematic biopsies and DRE guided biopsies revealed prostate cancer in all 17 cases. DRE guided biopsies did not increase the detection rate of systematic biopsies in this group.

Conclusion: Digitally guided biopsies additionally taken to systematic 12-core biopsies do not enhance the detection rate of prostate cancer in the patients with a PSA level greater than $20 \mathrm{ng} / \mathrm{ml}$, possibly due to a large volume disease, however, it may be a useful adjunct for the patients with a PSA level less than $10 \mathrm{ng} / \mathrm{ml}$ as well as in the presence of undifferentiated tumors.

Keywords: Prostate cancer; Tumors; 12-core Systematic biopsies 
Abbreviations: TRUSG: Transrectal Ultrasound Guided; PSA: Prostate Specific Antigen; DRE: Digital Rectal Examination.

\section{Introduction}

Prostate cancer has been the most common cancer affecting men in the USA, and the second leading cause responsible for cancer deaths [1]. Mortality can only be decreased by early diagnosis of organ confined cancer. Transrectal ultrasound guided (TRUSG) biopsies are usually indicated when the prostate specific antigen (PSA) level is elevated, and/or an abnormal digital rectal examination (DRE) finding exists. Low sensitivity and specificity of both PSA and DRE have led a low diagnostic yield of initial TRUSG biopsies, so that repeat biopsies are frequently required. Moreover, there has been no universally accepted biopsy protocol.

The operator dependent nature of DRE may lead to underestimation of its role in terms of specificity for cancer detection. Therefore, we conducted a clinical prospective study combining standard 12-core systematic TRUS guided biopsies and 2 additional cores under DRE guidance in case of suspicious DRE, to further elucidate significance of DRE properly performed by an experienced urologist.

\section{Materials and Methods}

A total of 119 consecutive patients who either have a PSA level greater than $4 \mathrm{ng} / \mathrm{ml}$ and/or suspicious DRE findings underwent standard 12 core systematic TRUS guided biopsies and 2 additional cores obtained under the finger guidance in DRE + cases. Patients were divided into three groups according to their PSA level. Group I $(n=8)$ : PSA <4ng/ml, Group II: ( $\mathrm{n}=62)$ PSA 4-10ng/ml, Group III $(\mathrm{n}=49):$ PSA $>10 \mathrm{ng} / \mathrm{ml}$. The patients whose pathology results were benign and have had no abnormalities on prostate were excluded from the study. Then the cancer detection ratios of both modalities were obtained according to the DRE abnormal patients.

\section{The Biopsy Protocol}

An informed consent was obtained. Anticoagulants were stopped at least three days before, however low dose aspirin was not an absolute contraindication. Urinary infection is excluded via urinalysis and urine culture. Prophylactic ciprofloxacin (500-750 mg) and metronidazole $(500 \mathrm{mg})$ were started 3 days prior to the procedure twice a day. Cleansing enema was performed 2 hours before. The patient was placed in left lateral decubitus position. Under light sedation with midazolam $0.03 \mathrm{mg} / \mathrm{kg}$, a through DRE is performed. Suspicious locations are noted for subsequent DRE guided additional biopsies. Povidone-iodine solution with lidocaine jelly was also inserted into the rectum prior to probe insertion. A B-mode frequency $4-9 \mathrm{MHz}$ transrectal probe with biopsy attachment was introduced. Twelve cores starting from apex lateral and mid portions followed by middle and basal far lateral and lateral biopsies on both sides. Depending upon the number of suspicious nodules on DRE, two or more DRE guided biopsies were also taken and numbered accordingly (Figure 1). The specimens were put in the numbered containers filled with $10 \%$ formalin. Patients were discharged on the same day, on three days of anti-inflammatory and antimicrobial prophylaxis. The complications were classified as minor or major if present.

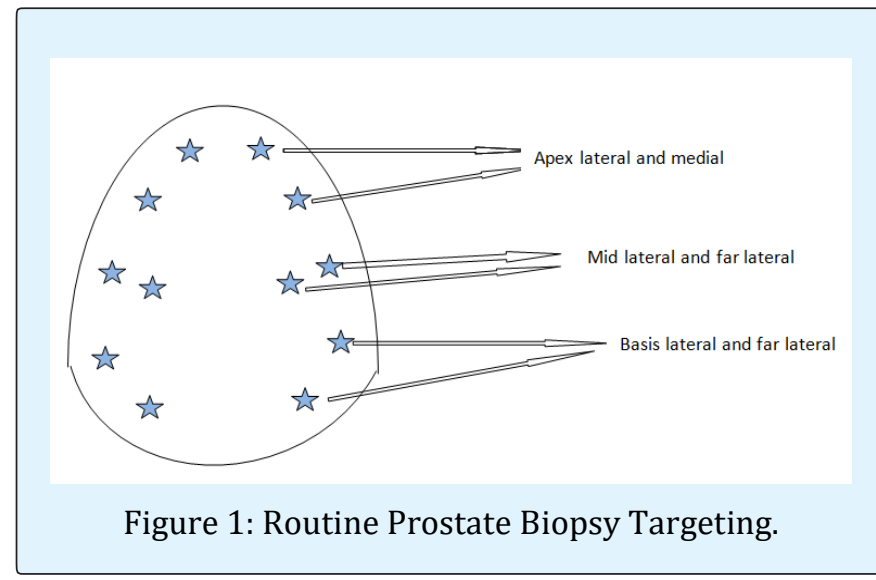

Biopsy proven cancers were regarded as true positives. Sensitivity, specificity, positive predictive value, negative predictive value and accuracy of each method were calculated in each group separately and compared to DRE and TRUS guided combined biopsies and $\mathrm{P}<0.05$ is considered significant.

\section{Results}

The distribution of groups and DRE and biopsy findings are represented in Table 1. First group had a PSA level less than $4 \mathrm{ng} / \mathrm{ml}$, they would have been missed without DRE examination. In the first group there were 3 patients with abnormal DRE findings, and 2 of 3 were detected as cancer with TRUSG standard biopsies. The biopsies additionally taken with DRE did not avail to find cancer different from TRUSG biopsies. 


\begin{tabular}{|c|c|c|c|c|c|}
\hline & $\begin{array}{c}\text { Number of } \\
\text { patients }\end{array}$ & Abnormal DRE & $\begin{array}{c}\text { Ca detected via TRUSG } \\
\text { biopsies }\end{array}$ & $\begin{array}{c}\text { Additional biopsies } \\
\text { detected cancer }\end{array}$ & $\begin{array}{c}\text { Total cancer } \\
\text { detected }\end{array}$ \\
\hline Group I & \multirow{2}{*}{8} & \multirow{2}{*}{3} & \multirow{2}{*}{2} & \multirow{2}{*}{1} & \multirow{2}{*}{2} \\
\hline $\mathrm{PSA}<4 \mathrm{ng} / \mathrm{ml}$ & & & & & \\
\hline Group II & \multirow{2}{*}{62} & \multirow{2}{*}{10} & \multirow{2}{*}{7} & \multirow{2}{*}{$6^{*}$} & \multirow{2}{*}{8} \\
\hline PSA 4-10 $\mathrm{ng} / \mathrm{ml}$ & & & & & \\
\hline Group III & \multirow{2}{*}{49} & \multirow{2}{*}{22} & \multirow{2}{*}{17} & \multirow{2}{*}{17} & \multirow{2}{*}{17} \\
\hline $\mathrm{PSA}>4 \mathrm{ng} / \mathrm{ml}$ & & & & & \\
\hline Total & 119 & 35 & 26 & 24 & 27 \\
\hline
\end{tabular}

Table 1: The distribution of groups.

One of the detected cancers was different from those which were taken by TRUSG biopsies (Table 2).

\begin{tabular}{|c|c|c|c|}
\hline Group I (n: 3) & Additional Bx & Standard 12- Core Biopsy & Total Ca Detected \\
\hline PSA<4 ng/ml + DRE abnormal & 1 & 2 & 2 \\
\hline No of biopsy detected cancer & 2 & 1 & - \\
\hline No of biopsy not detected cancer & 2 & 1 & \\
\hline
\end{tabular}

Table 2: The numbers of TRUS and DRE guided biopsy cancers in Group I are shown with regards to DRE positivity.

The group II had 1 cancer only detected by DRE guided biopsies (p:0,4) (Table 3).

\begin{tabular}{|c|c|c|c|}
\hline Group II (n: 10) & \multirow{2}{*}{ Additional Bx } & Standard 12- Core Biopsy & Total Ca detected \\
\cline { 1 - 2 } PSA 4-10 ng/ml +DRE abnormal & 6 & 7 & 8 \\
\hline No of biopsy detected cancer & 2 & 1 & - \\
\hline No of biopsy not detected cancer & 2 & 7 & - \\
\hline
\end{tabular}

Table 3: The numbers of TRUS and DRE guided biopsy cancers in Group II are shown with regards to DRE positivity.

In the third group standard TRUS guided systematic 12-core biopsies were able to detect all the cancers regardless of DRE abnormality (Table 4).

\begin{tabular}{|c|c|c|c|}
\hline Group III (n: 22) & \multirow{2}{*}{ Additional Bx } & Standard 12-Core Biopsy & Total Ca detected \\
\cline { 1 - 4 } PSA >10 ng/ml +DRE abnormal & 17 & 17 & 17 \\
\hline No of biopsy detected cancer & 5 & 5 & - \\
\hline No of biopsy not detected cancer & & 5 & \\
\hline
\end{tabular}

Table 4: The numbers of TRUS and DRE guided biopsy cancers in Group III are shown with regards to DRE positivity.

Overall the biopsies additionally taken with DRE helped to be diagnosed 1 of the 27 cases. No major complications were seen. Hematuria and hemospermia were among the transient minor complications.

\section{Discussion}

TRUS guided biopsies are the only way for definitive diagnosis of prostate cancer. It is of utmost importance to detect the cancer in the initial biopsy if it exists. Thereby, the patients would have a better chance for cure, on the other hand will be saved from unnecessary repeated biopsies. Six quadrant biopsies had been accepted as the gold standard, however, it is associated with a relatively high false-negative rate of $15 \%$ to $31 \%$ [2,3]. Extensive biopsies particularly from far lateral aspects of the gland yielded higher detection rates [4].

Although there is no universal agreement upon the biopsy protocol, any abnormality during DRE examination and/ or elevated PSA level are general indications for prostate biopsy. Currently, the majority of the prostate biopsies turn out to be negative either because of low specificity of PSA and DRE or low detection rate of biopsy 
itself. In order to avoid unnecessary biopsies, age, \%fPSA, PSA density, PSA velocity, prostate health index (phi) were proposed [5]. On the other hand, various attempts have been made to increase the detection rate of TRUSG biopsies such as saturation biopsies more than 20 obtained through the perineum, in accordance with the prostate volume. However, the complication rate also increases as the number of cores, particularly in younger patients.

MRI guided biopsies, MRI and TRUS fusion techniques are not readily available and cost-effective currently in daily practice, so that we have to continue to use the same tools unless proved otherwise. Since both radiologists and urologists have been performing TRUS guided biopsies, a proper DRE may be underestimated oftentimes. DRE is not usually sensitive for the tumors less than one centimeter [6]. It has been shown that if the initial biopsies are negative in the patients with a PSA level greater than $20 \mathrm{ng} / \mathrm{ml}$, second biopsy has a substantial chance for the diagnosis of prostate cancer [7].

Prakash and colleagues compared 10 cores versus 16 cores in DRE + cases without targeted biopsies. Although they found a significantly increased detection rate with 16 core biopsies, additional DRE targeted biopsies would probably enhance the detection rate in this group similarly, without a remarkable increase in the core number [8].

In this study, we showed a small percentage, but highly aggressive cancers may appear without PSA elevation, but only an abnormality can be found during a DRE before the disease is disseminated. In the first group, 3 patients were diagnosed solely based on DRE findings and subsequent biopsies, which in turn, revealed poorly differentiated high grade prostate cancer. Likewise, in the second group of the study, 1 out of 7 cancers was found only in DRE guided biopsy specimens.

Overall, DRE and TRUS guided systematic biopsy combination significantly enhanced the detection rate. TRUS evaluation of the prostate is of limited value; however, hypoechoic lesions should also be targeted, considering the fact that one third of them would harbor malignant tissue. In the study, hypoechoic lesions were also targeted and the number included in the systematic biopsies. Since DRE examination requires experience and expertise, it was our main point of interest. One of the drawbacks of 2 D TRUS evaluation is trajectories of the samples may not be visualized, therefore accurate mapping of the prostate may not be possible. Three dimensional TRUS guided biopsies provide tracking of each sample location and precise mapping, hence lessens duplicated biopsies as may be seen with two dimensional TRUS imaging [9].

In the Group III, DRE guided biopsies did not do any better compared to systematic biopsies. This may be explained that the higher the PSA is, the more prostate is involved in the disease, which increases the chance of being detected. However, due to our limited number of the patients in this group, this does not necessarily mean DRE is not important if the PSA level is $>10 \mathrm{ng} / \mathrm{ml}$. We will continue to add DRE guided biopsies regardless of the PSA level when necessary.

Multiparametric MRI has become a very widely used tool for the detection of prostate cancer in the last decade. The fusion biopsy techniques have promising results especially for individually targeting biopsies rather than using a standart biopsy scheme [10].

The main limitation of this study is the small sample size. The operator dependent nature of DRE makes it prone to be interobserver variability even among the urologists. We suggest a thorough evaluation by DRE of each and every patient to better keep our patients out of the harm's way.

\section{References}

1. Siegel R, Ma J, Zou Z, Jemal A (2014) Cancer Statistics, 2014. CA CANCER J CLIN 64(1): 9-29.

2. Singh H, Canto EI, Shariat SF, Kadmon D, Miles BJ, et al. (2004) Improved detection of clinically significant, curable prostate cancer with systematic 12-core biopsy. J Urol 171(3): 1089-1092.

3. Durkan GC, Sheikh N, Johnson P, Hildreth AJ, Greene DR (2002) Improving prostate cancer detection with an extended-core transrectal ultrasonography-guided prostate biopsy protocol. BJU Int 89(1): 33-39.

4. de la Taille A, Antiphon P, Salomon L, Cherfan M, Porcher R, et al. (2003) Prospective evaluation of a 21-sample needle biopsy procedure designed to improve the prostate cancer detection rate. Urology 61(6): 1181-1186.

5. Chadou H, Fetati H, Boulerial A, Keroumi, Boudia F, et al. (2018) Application of PSA Kinetics on Prostate Cancer: Experience of Pharmacovigilance Department of University Hospital Establishment of ORAN (UHE) 


\section{Open Access Journal of Urology \& Nephrology}

in Algeria. Open Access Journal of Urology and Nephrology 3(3): 1-6.

6. Presti JC Jr, O’Dowd GJ, Miller MC, Mattu R, Veltri RW (2003) Extended peripheral zone biopsy schemes increase cancer detection rates and minimize the variance in prostate specific antigen and age related cancer rates: results of a community multi-practice study. J Urol 169(1): 125-129.

7. Ravizzini G, Turkbey B, Kurdziel K, Choyke PL (2009) New Horizons in Prostate Cancer Imaging. Eur J Radiol 70(2): 212-226.

8. Shaida N, Jones C, Ravindranath N, Malone PR (2009) The Chances of Subsequent Cancer Detection in Patients with a PSA $>20 \mathrm{ng} / \mathrm{ml}$ and an Initial Negative Biopsy. Scientific World Journal 9: 343-348.
9. Prakash VS, Mohan GC, Krishnaiah SV, Vijaykumar V, Babu GR, et al. (2013) Ten-core versus 16-core transrectal ultrasonography guided prostate biopsy for detection of prostatic carcinoma: a prospective comparative study in Indian population. Prostate Int 1(4): 163-168.

10. Schoots IG, Roobol MJ, Nieboer D, Bangma CH, Steyerberg EW, et al. (2015) Magnetic resonance imaging-targeted biopsy may enhance the diagnostic accuracy of significant prostate cancer detection compared to standard transrectal ultrasound-guided biopsy: a systematic review and meta-analysis. Eur Urol 68(3): 438-450. 